\title{
USO DE TECNOLOGIAS NO ENSINO DE SEMIOLOGIA E SEMIOTÉCNICA DE ENFERMAGEM
}

\author{
USE OF TECHNOLOGY IN THE TEACHING OF \\ NURSING SEMIOLOGY AND SEMIOTECHNIQUE
}

\section{USO DE LA TECNOLOGÍA EN LA ENSEÑANZA DE SEMIOLOGÍA Y SEMIOTÉCNICA DE ENFERMERÍA}

\author{
Evandro Bernardino Mendes de Melo ${ }^{1}$ \\ Marcio Coutinho Farias Leite ${ }^{2}$ \\ Filipe Martinuzo Filetti ${ }^{3}$ \\ Elizabete Regina Araújo de Oliveira ${ }^{4}$ \\ Lorena Barros Furieri ${ }^{5}$ \\ Mirian Fioresi ${ }^{6}$
}

Como citar este artigo: Melo EBM, Leite MCF, Filetti FM, Oliveira ERA, Furieri LB, Fioresi M. Uso de tecnologias no ensino de semiologia e semiotécnica de enfermagem. Rev baiana enferm. 2019;33:e33859.

Objetivo: relatar a vivência de discentes, docentes e monitores na utilização de diferentes tecnologias educativas durante ensino teórico-prático de semiologia e semiotécnica de enfermagem. Método: relato de prática de ensino de 2 monitores, 2 docentes e 187 alunos de um curso de graduação em enfermagem de Vitória, Espírito Santo, realizado de 2015 a 2017, com reflexões sustentadas no referencial teórico de Merhy. Resultados: o ensino foi mediado por tecnologias leves associadas às leve-duras e duras, buscando-se o desenvolvimento de competências que pudessem aprimorar a produção de conhecimentos e que possam futuramente ser aplicadas na prática profissional, tendo como horizonte um cuidado de enfermagem humanizado e não restrito ao tecnicismo. Conclusão: a práxis pedagógica relatada favoreceu ao desenvolvimento de competências relacionais e manuais, à autonomia discente e ao pensamento crítico-reflexivo, necessários à articulação teórico-prática, à tomada de decisão clínica e a novos modos de se produzir cuidado em enfermagem.

Descritores: Educação em Enfermagem. Inovação. Tecnologia Educacional. Ensino. Enfermagem.

Objective: to report the experience of students, professors and professor's assistants (PA) when using different educational technologies during theoretical-practical teaching of nursing semiology and semiotechnique. Method: report of teaching practice of 2 PA, 2 professors and 187 students from a Nursing graduate course in Vitória, Espirito Santo, conducted from 2015 to 2017, with reflections based on Merhy's theory. Results: the teaching was mediated by soft technologies associated to soft-hard ones, seeking the development of skills that could enhance knowledge production, and, in the future, be applied in professional practice, having as borizon a bumanized nursing care, not restricted to techniques. Conclusion: the reported pedagogical praxis favored the development of relational and manual competences, the student's autonomy and critical and reflective thinking, necessary the theoretical-practical articulation, clinical decision-making and new ways of producing nursing care.

Descriptors: Education, Nursing. Innovation. Educational Technology. Teaching. Nursing.

\footnotetext{
Enfermeiro. Mestre em Enfermagem. Universidade Federal de Minas Gerais. Professor da Católica de Vitória Centro Universitário. Vitória, Espírito Santo, Brasil. evandromendes20@yahoo.com.br. http://orcid.org/0000-0002-1772-3083

Enfermeiro. Pesquisador Independente. Vitória, Espírito Santo, Brasil. http://orcid.org/0000-000 I-5392-0968

Enfermeiro. Mestre em Fisiologia. Universidade Federal do Espírito Santo. http://orcid.org/0000-0002-8|4|-4007.

Enfermeira. Doutora em Enfermagem. Professora do Departamento de Enfermagem da Universidade Federal do Espírito Santo. http://orcid.org/0000-0002-66 I6-4273

Enfermeira. Doutora em Fisiologia. Professora da Universidade Federal do Espírito Santo. Vitória, Espírito Santo, Brasil. http://orcid.org/0000-0002-8560-4385

Enfermeira. Doutora em Fisiologia. Professora do Departamento de Enfermagem da Universidade Federal do Espírito Santo. http://orcid.org/0000-0003-3859-2227
} 
Objetivo: reportar la experiencia de los estudiantes, profesores y monitores en el uso de diferentes tecnologías educativas durante la enseñanza teórica-práctica de semiología y semiotécnica de enfermería. Método: relato de práctica docente de 2 monitores, 2 profesores y 187 estudiantes de un curso de graduación en enfermería de Vitória, Espirito Santo, realizado de 2015 a 2017, con reflexiones sostenidas sobre el referencial teórico de Merhy. Resultados: la enseñanza era mediada por tecnologías leves, asociadas a leves-duras, buscando el desarrollo de habilidades que podrían mejorar la producción de conocimiento, y que, en el futuro, podrían ser aplicadas en la práctica profesional, teniendo como horizonte un cuidado de enfermería bumanizado y no restringido al tecnicismo. Conclusión: la praxis pedagógica relatada favoreció el desarrollo de competencias relacionales y manuales, la autonomía de los estudiantes y el pensamiento crítico y reflexivo, necesarios para la vinculación teórico-práctica, la toma de decisiones clinicas y las nuevas formas de producción de los cuidados de enfermería.

Descriptores: Educación en Enfermería. Innovación. Tecnología Educacional. Enseñanza. La Enfermería.

\section{Introdução}

O principal objetivo da formação acadêmica em enfermagem é diplomar enfermeiros qualificados, capazes de cuidar de pacientes em ambientes clínicos, com habilidade de tomada de decisões. Assim, o ensino de enfermagem deve concentrar-se em métodos que incentivem habilidades de resolução de problemas e desenvolvimento de pensamento crítico ${ }^{(1)}$.

Nesse sentido, os cursos de graduação em enfermagem incluem, em seus currículos, a disciplina de semiologia e semiotécnica, necessária à construção de conhecimentos que possibilitem a realização do cuidado nos diversos ambientes em que o profissional de enfermagem insere-se. O ensino dessa disciplina materializa-se por meio de atividades teórico-práticas, fundamentais para disparar a reflexão crítica, promover a apreensão de conceitos-base e o desenvolvimento de habilidades e competências com base em situações simuladas ou reais de cuidado ${ }^{(2)}$.

Essa formação torna o enfermeiro capaz de realizar uma satisfatória coleta de dados, primeira etapa do Processo de Enfermagem (PE), na qual são reunidos dados subjetivos e objetivos do cliente, por meio de anamnese e exame físico, com a finalidade de obter informações que subsidiem o registro, o planejamento e a implementação de condutas com o paciente de forma sistematizada ${ }^{(3-4)}$.

O ambiente/momento de ensino da semiologia e semiotécnica deve ser potencializado pelo uso de diferentes métodos que favoreçam a aprendizagem e contribuam para processos pedagógicos inovadores e eficazes. Uma estratégia de ensino-aprendizagem comumente utilizada na área da saúde é a monitoria, serviço de apoio pedagógico que tem a capacidade de estimular a autonomia discente e promover um canal de diálogo entre alunos e professores ${ }^{(5)}$. O uso de diferentes tecnologias como instrumentos pedagógicos também é um grande aliado ao aprendizado em enfermagem, pois potencializa o desenvolvimento de um processo pedagógico que supere modos de ensinar em semiologia e semiotécnica centrados exclusivamente em técnicas.

O trabalho em saúde é relacional e, portanto, traz como centralidade o trabalho vivo, isto é, os produtos do cuidado em saúde realizam-se em ato. Assim, o ato de cuidar implica necessariamente o uso de tecnologias de saúde leves (que englobam as relações interpessoais, o vínculo e o acolhimento), as quais devem ser intercambiadas com as tecnologias leve-duras (que agregam o conhecimento estruturado e sustentado nas Ciências da Saúde) e duras (relativas aos equipamentos e dispositivos). É a interação das diferentes tecnologias no ato de cuidar que conforma o processo de trabalho em saúde, que pode ser mais rígido e protocolar ou mais dinâmico e dialógico ${ }^{(6)}$.

Aplicando-se esta reflexão no contexto da práxis pedagógica, compreende-se que diferentes tecnologias podem ser utilizadas no contexto do ensino-aprendizagem de enfermagem, para promover o desenvolvimento de 
habilidades que permitam que os futuros enfermeiros produzam um trabalho vivo em ato ${ }^{(6)}$ criativo e, sobretudo, centrado mais nas relações que nos instrumentais.

Pelo exposto, este estudo objetivou relatar a vivência de discentes, docentes e monitores na utilização de diferentes tecnologias educativas durante o ensino teórico-prático de semiologia e semiotécnica de enfermagem.

\section{Método}

Trata-se de estudo descritivo, do tipo relato de experiência, produto das vivências práticas de professores, monitores e discentes de um curso de graduação em enfermagem de uma universidade pública de Vitória, Espírito Santo. Os resultados refletem as discussões sobre as estratégias de intervenção metodológicas realizadas entre março de 2015 e dezembro de 2017, durante as aulas teórico-práticas e os momentos de monitoria da disciplina de semiologia e semiotécnica. Os participantes das atividades apresentadas neste relato foram 2 professores, 2 monitores e 187 estudantes (em média, 31 graduandos por semestre).

A disciplina teórico-prática de semiologia e semiotécnica é obrigatória ao $3^{\circ}$ período do curso de graduação cenário deste estudo. Tem carga horária total de 120 horas e ainda prevê 20 horas mensais de monitoria. Segundo seu projeto pedagógico, busca preparar o aluno para a compreensão da semiologia e semiotécnica como parte do PE, direcionando suas atividades na perspectiva integral do ser humano. Ao final da disciplina, espera-se que os acadêmicos saibam caracterizar as alterações fisiológicas, baseando-se nas funções dos sistemas e estruturas do corpo humano, interpretando dados objetivos e subjetivos para a realização do diagnóstico de enfermagem, segundo a NANDA Internacional, Inc. (NANDA-I $)^{(7)}$, as intervenções de acordo com a Nursing Interventions Classifications (NIC) ${ }^{(8)}$ e a aplicação das demais etapas do PE. Espera-se, ainda, que o estudante seja apto a distinguir o exame físico de enfermagem da anamnese aplicada por outras profissões de saúde, e que este seja realizado conforme o referencial teórico adotado pela disciplina.

Inicialmente, os conteúdos abrangem os temas de biossegurança, marketing pessoal, legislação e PE; em seguida, o ensino centra-se nos tópicos relacionados ao exame físico, que são trabalhados segundo a teoria das Necessidades Humanas Básicas (NHB) de Wanda Aguiar Horta. Com base nessa abordagem, são discutidos os conteúdos e as técnicas relacionadas às avaliação das necessidades psicossociais e psicobiológicas do ser humano, que envolvem: integridade cutaneomucosa, cuidado corporal e nutrição; percepção olfatória, gustativa, visual e auditiva; regulação vascular, oxigenação e crescimento celular; eliminação, regulação hidrossalina e eletrolítica; regulação neurológica; mecânica corporal, exercício, atividade física e locomoção; e sexualidade ${ }^{(9)}$.

Após as aulas de cunho mais teórico acerca de um tema, as monitorias são operacionalizadas no laboratório de técnicas de enfermagem, nos dias que sucedem as aulas teórico-práticas (ministradas em sala e também no laboratório), e instantes antes de os alunos irem para a prática em um hospital universitário, possibilitando ao estudante resgatar os conhecimentos construídos junto com os professores e, em seguida, realizar os procedimentos e as técnicas aprendidos, numa perspectiva holística. A participação nesses momentos é facultativa aos estudantes; contudo, cumpre destacar que há assiduidade de praticamente todos os matriculados. Acontecem em horários flexíveis, previamente agendados, de acordo com a necessidade dos alunos e a disponibilidade dos monitores e do laboratório de práticas. A biblioteca também é utilizada para a construção de instrumentos de coleta de dados.

A disciplina é conduzida por meio do uso de diferentes metodologias de ensino, tradicionais e ativas, que conjugam várias tecnologias mediadoras de aprendizagem, as quais estão sistematizadas neste relato, com base em uma análise crítica sustentada pela proposta classificatória de Merhy para as tecnologias de saúde: leves, leve-duras e duras ${ }^{(6)}$. Busca-se tecer uma reflexão 
sobre novos modos de ensinar que, por consequência, conduzirão a novos modos de pensar e fazer saúde.

Esclarece-se que, em se tratando de um relato de experiência, o projeto não foi submetido a Comitê de Ética em Pesquisa; entretanto, este estudo obedeceu aos princípios da bioética em conformidade com as Resoluções n. 466/2012 e n. 510/2016 do Conselho Nacional de Saúde, incluindo a utilização de estratégias para assegurar o anonimato dos participantes das ações educacionais descritas.

\section{Resultados e Discussão}

A combinação de atividades teóricas e práticas é fundamental na formação do profissional de enfermagem para o desenvolvimento de habilidades técnicas, postura ética e capacidade analítico-crítico-reflexiva. Potencializam, portanto, a consolidação do aprendizado e a construção de novos saberes. Nesse processo, a disciplina de semiologia e semiotécnica tem papel relevante, porque inaugura a aproximação dos estudantes à realidade profissional e aos instrumentais e rotinas do trabalho da enfermagem ${ }^{(10)}$.

Assim, no contexto deste relato, a utilização de tecnologias leves, leve-duras e duras esteve presente em todos os processos de ensino da semiologia e semiotécnica, desde o contato inicial entre docente, monitor e estudantes até o fim do período letivo, conforme descreve o Quadro 1.

Quadro 1 - Tecnologias e contexto de sua utilização nas aulas teórico-práticas e momentos de monitoria de semiologia e semiotécnica em enfermagem

\begin{tabular}{|c|c|c|c|}
\hline Tecnologias & Classificação & Materiais e ambiente & Descrição do processo \\
\hline Acolhimento & Leve & Laboratório de práticas & $\begin{array}{l}\text { Momento inicial da disciplina, } \\
\text { com a apresentação dos docentes, } \\
\text { monitores, dinâmica das aulas e dos } \\
\text { momentos de monitoria, e respostas } \\
\text { a possíveis dúvidas relacionadas à } \\
\text { disciplina. Esta tecnologia é também } \\
\text { utilizada durante todo o semestre. }\end{array}$ \\
\hline $\begin{array}{l}\text { Práticas em } \\
\text { dupla }\end{array}$ & Leve-dura & $\begin{array}{l}\text { Desenvolvido no } \\
\text { laboratório de práticas, } \\
\text { com uso de diversos } \\
\text { materiais, dependendo dos } \\
\text { conteúdos e das técnicas } \\
\text { abordadas. Como exemplo, } \\
\text { citam-se o estetoscópio e } \\
\text { o esfigmomanômetro. }\end{array}$ & $\begin{array}{l}\text { Simulação das técnicas nos colegas. } \\
\text { Exemplo: ao praticar os sinais vitais, } \\
\text { um aluno simula ser o paciente } \\
\text { enquanto o outro incorpora o papel } \\
\text { do enfermeiro. }\end{array}$ \\
\hline $\begin{array}{l}\text { Construção } \\
\text { de estudos } \\
\text { de caso e de } \\
\text { instrumentos de } \\
\text { coleta de dados }\end{array}$ & Leve-dura & $\begin{array}{l}\text { Desenvolvido no } \\
\text { Hospital, laboratório } \\
\text { de práticas e biblioteca. } \\
\text { Utiliza-se a NANDA e } \\
\text { o Nursing Interventions } \\
\text { Classifications. }\end{array}$ & $\begin{array}{l}\text { Coleta de dados desenvolvida no } \\
\text { hospital, realiza-se um estudo de } \\
\text { caso semanal. }\end{array}$ \\
\hline Cartaz/pôster & Leve-dura & $\begin{array}{l}\text { Papel cenário ou cartolina, } \\
\text { pincel, papel ofício, } \\
\text { impressora. }\end{array}$ & $\begin{array}{l}\text { Confecciona-se pôster para } \\
\text { ilustração da avaliação dos pares de } \\
\text { nervos cranianos. }\end{array}$ \\
\hline Manequins & Dura & $\begin{array}{l}\text { Desenvolvido no } \\
\text { laboratório de práticas, } \\
\text { com utilização de } \\
\text { manequim, caixa de som } \\
\text { e fones de ouvido. }\end{array}$ & $\begin{array}{l}\text { Realiza-se a ausculta dos sons } \\
\text { cardíacos e pulmonares. }\end{array}$ \\
\hline
\end{tabular}


Quadro 1 - Tecnologias e contexto de sua utilização nas aulas teórico-práticas e momentos de monitoria de semiologia e semiotécnica em enfermagem

\begin{tabular}{|l|l|l|l|}
\hline \multicolumn{1}{|c|}{ Tecnologias } & Classificação & \multicolumn{1}{|c|}{ Materiais e ambiente } & \multicolumn{1}{c|}{ Descrição do processo } \\
\hline $\begin{array}{l}\text { Materiais } \\
\text { clínicos }\end{array}$ & Dura & $\begin{array}{l}\text { Martelo clínico, lanterna } \\
\text { clínica, oftalmoscópio, } \\
\text { otoscópio, estetoscópio, } \\
\text { termômetro, balança, } \\
\text { espéculo nasal. } \\
\text { Desenvolvido no } \\
\text { laboratório de práticas e } \\
\text { no hospital. }\end{array}$ & $\begin{array}{l}\text { Utilização e manuseio dos } \\
\text { equipamentos de forma } \\
\text { adequada. Exemplo: regulagem } \\
\text { e posicionamento de um } \\
\text { oftalmoscópio. }\end{array}$ \\
\hline $\begin{array}{l}\text { Aplicativos para } \\
\text { dispositivo } \\
\text { móvel }\end{array}$ & Dura & $\begin{array}{l}\text { Utilizado em todos os } \\
\text { ambientes de ensino, em } \\
\text { modo online ou offline. }\end{array}$ & $\begin{array}{l}\text { Softwares com a temática de } \\
\text { semiologia ou temas relacionados. }\end{array}$ \\
\hline
\end{tabular}

Fonte: Elaboração própria.

Conforme explicitado no Quadro 1, o acolhimento inicial - tecnologia leve - ocorreu no laboratório de técnicas, cujas cadeiras foram organizadas no formato de círculo, para facilitar o diálogo entre os participantes. Foi realizado no primeiro contato com as turmas de cada semestre, com o apoio de diferentes dinâmicas. Uma abordagem adotada, por exemplo, foi o compartilhamento das vivências na disciplina pela turma do período anterior, o que possibilitou à nova turma a expressão de suas dúvidas, medos, expectativas e curiosidades em relação à disciplina. Nesse momento também se iniciou a formação de vínculo entre os alunos, os professores e os monitores e pactuaram-se as formas de comunicação. Os monitores colocaram-se à disposição, bem como explicaram as dinâmicas de funcionamento da disciplina, as regras de utilização do laboratório e as práticas desenvolvidas no ambiente hospitalar que, para a maioria dos alunos, é um ambiente novo.

O acolhimento, na perspectiva do vínculo, permeou todo o período letivo, em modo coletivo, mas também individual, quando os docentes e/ou monitores perceberam essa necessidade, em especial quando os alunos demonstraram ansiedade e medo nos momentos que antecederam a abordagem ao paciente. Nestes casos, os docentes ou monitores atuaram como intermediários entre o estudante e o paciente, transmitindo-lhe confiança e estimulando a sua proatividade.
O vínculo entre docente, monitor e alunos foi imprescindível para a diminuição do nervosismo dos alunos que, pela primeira vez, tiverem contato direto com pacientes no cenário hospitalar. Para isso, as dinâmicas de acolhimento foram basilares para a garantia do suporte emocional aos estudantes. Evidências mostram que a ausência desse tipo de apoio pode levar os estudantes a experienciarem solidão social e emocional ${ }^{(11)}$.

Outra tecnologia leve que permeou toda a disciplina foi a comunicação interpessoal, que não se restringiu aos ambientes acadêmicos. Aplicativos de mensagens foram utilizados para facilitar o contato entre monitores e alunos. Os professores também promoveram momentos de descontração antes das avaliações práticas, como a aplicação de técnicas de relaxamento. Um momento importante, no qual o uso das tecnologias leves provou seu valor, foi quando, instantes antes de uma avaliação, uma aluna disse ao monitor que precisava apenas de um abraço para sentir-se mais calma.

Quanto às tecnologias leve-duras, na disciplina foram trabalhadas as práticas executadas em duplas, a construção de cartazes e de roteiros de exame físico e de estudos de casos, com base na taxonomia NANDA- $\mathrm{I}^{(7)}$ e NIC ${ }^{(8)}$. Tais atividades foram desenvolvidas no laboratório, no hospital e na biblioteca.

As dinâmicas em dupla foram efetivadas no laboratório, em sincronia ou após a demonstração 
prática do conteúdo e/ou técnicas pelo professor. Ao realizarem a prática semiológica com os próprios colegas, os acadêmicos representavam ora o paciente, ora o enfermeiro, em um sistema de revezamento de duplas, permitindo, assim, melhorar a percepção da prática adequada. Ao trabalhar-se com alguns conteúdos específicos, também se fez necessário o uso de diferentes tecnologias duras, como manequins com sons para auscultas cardíaca e respiratória.

As práticas em dupla exigiram que o estudante resgatasse os saberes construídos durante as aulas e começasse a desenvolver as suas habilidades semiológicas. Para tanto, o acadêmico precisou ser proativo, observador e ouvinte e comunicar-se com os colegas de forma dialógica, acionando, portanto, suas competências relacionais.

A tecnologia cartaz foi utilizada para a apresentação e teste de funcionalidade dos pares de nervos cranianos. Confeccionado pelos monitores sob a supervisão dos professores e afixado em local de fácil acesso, foi bastante consultado pelos estudantes. A elaboração dos roteiros para a realização do exame físico iniciou-se após as aulas teóricas e finalizou no decorrer das aulas práticas, de acordo com o raciocínio do estudante, que estava livre para acrescentar e redigir o roteiro de acordo com as suas necessidades. Quando surgiam dúvidas, o monitor era consultado e indicava possíveis melhorias. Esses roteiros foram utilizados tanto no hospital quanto no laboratório ou outro ambiente que o estudante estivesse disponível para praticar.

Ao utilizarem os recursos citados anteriormente no laboratório, os estudantes passaram a perceber as necessidades do paciente de forma mais clara quando iniciavam as atividades no hospital. Munidos dessa prática, eles conseguiram desenvolver estudos de caso semanais, os quais foram elaborados com base nos dados objetivos e subjetivos coletados durante o histórico de enfermagem e o exame físico. Com base neles foram propostos diagnósticos e intervenções de enfermagem, conforme a taxonomia da NANDA-I ${ }^{(7)}$ e da NIC, respectivamente ${ }^{(8)}$. Todos os estudos foram corrigidos e um feedback foi dado a cada estudante, para que pudesse perceber os pontos que precisavam ser aperfeiçoados. A construção dos estudos de caso requereu autonomia do estudante, associação dos diferentes conhecimentos adquiridos nas diferentes disciplinas que já cursou e o raciocínio clínico, com vistas à tomada de decisões assertivas ${ }^{(12)}$.

As tecnologias duras utilizadas foram: manequins para ausculta cardíaca e pulmonar, instrumentos clínicos e uso de aplicativos para dispositivos móveis. Com o uso do manequim de ausculta cardíaca e pulmonar, os estudantes conseguiram diferenciar os sons fisiológicos e suas alterações. Essa atividade pôde ser realizada tanto de forma individual quanto em grupos, e os monitores e docentes atuaram como facilitadores. Nesses momentos, os alunos tiveram a oportunidade de sanar as suas dúvidas e conseguiram perceber o que esperar dos pacientes de acordo com o seu estado de saúde. Nos primeiros contatos com essa tecnologia, os alunos, em geral, demonstraram dificuldade em identificar e/ou diferenciar os sons cardíacos e pulmonares; quando em contato com o paciente no ambiente hospitalar, no entanto, eles mostraram-se empolgados e hábeis na prática da ausculta.

Quanto aos instrumentos clínicos, foram manuseados e aplicados praticamente durante todo o decurso da disciplina, no laboratório e no hospital, para a realização do exame físico. Os alunos também aprenderam a calibrar as balanças para aferição de peso e receberam orientações sobre como cuidar dos equipamentos e armazená-los. Também foram utilizados aplicativos para dispositivo móvel sobre as temáticas relacionadas à semiologia e semiotécnica, cuja vantagem foi a possibilidade do seu uso no modo offline, em qualquer ambiente de aprendizado ou prática. Em momentos de dúvidas, os estudantes recorriam a esses recursos, alguns dos quais fornecem roteiros, sons para ausculta cardíaca e pulmonar, além de vídeos.

Extrapolando essa práxis pedagógica para o campo da saúde, relembra-se Merhy ${ }^{(6)}$, para o qual as tecnologias leves são relacionais e interativas. Ao relacioná-las às outras tecnologias 
- leve-duras e duras - produz-se um processo de trabalho que, à semelhança do trabalho em educação, resulta no trabalho vivo em ato, isto é, produz-se cuidado (ou conhecimento, no campo da educação) no exato momento em que o trabalho é executado. O trabalho, nesses dois campos, é sempre coletivo; assim, para cuidar/ensinar é preciso envolver o outro (colega de trabalho, aluno, monitor, colega de classe). Juntos, mediante o compartilhamento de suas tecnologias, opera-se o cuidado e constrói-se o conhecimento ${ }^{(6)}$.

Neste raciocínio, considera-se assertivo utilizar as tecnologias leves durante todo o decurso da disciplina, em intermédio com as leve-duras e duras, buscando sempre um contexto de acolhimento mútuo (professor-monitor-aluno) para a promoção de competências relacionais que possam aprimorar a produção de conhecimentos durante o ensino, mas que também possam futuramente ser aplicadas na prática profissional, tendo como horizonte um cuidado de enfermagem humanizado.

Este tipo de cuidado requer a efetiva apropriação das tecnologias leve-duras, as quais permitem o desenvolvimento de habilidades voltadas ao saber técnico estruturado, o PE por exemplo, que requer uma bagagem de conhecimentos para o julgamento clínico e, por conseguinte, para a produção de um modo diferente de cuidado, que seja centrado nas necessidades e subjetividade de cada indivíduo ${ }^{(6)}$. Requer ainda o uso consciente das tecnologias duras, para que o cuidado não se limite à rigidez das técnicas mecanicistas e, portanto, à conformação de um processo de trabalho morto.

Trabalho morto é aquele que é pregresso, já realizado e que, portanto, está incorporado aos instrumentos e equipamentos e dele não deriva um produto ${ }^{(6)}$. Então, o processo de trabalho em saúde, centrado nas tecnologias duras, resulta em um cuidado pouco flexível e consciente das demandas individuais. Assim, o processo de ensino-aprendizagem relatado aqui não se limitou ao rígido e prescritivo e nem à sobreposição das tecnologias duras sobre as relacionais, mas, ao contrário, permitiu que os alunos utilizassem diferentes tipos de tecnologias simultaneamente, refletindo e desmistificando a ideia de um ensino e de uma prática profissional em enfermagem tecnicista.

Viu-se, por exemplo, que as habilidades técnicas foram trabalhadas, sobretudo, com base na estratégia de simulação, após a troca de papéis em duplas (ora o estudante encenava o enfermeiro, ora, o paciente) e com uso de manequins. Essas técnicas, segundo a literatura, aprimoram não apenas a destreza manual, mas também as competências relacionais e fortalecem os processos cognitivos mobilizados para articular a teoria às situações reais de cuidado ${ }^{(13-15)}$. Fica claro, então, que as tecnologias leves são fundamentais para as relações de aprender a aprender e de aprender a cuidar.

O processo pedagógico, historicamente, tem se centrado na figura do professor e na transmissão de conhecimentos que devem ser memorizados pelos estudantes. Contudo, esse modelo de ensino já se provou ineficaz, já que relega o aluno a um papel passivo que pouco contribui para a apreensão de saberes. Na tentativa de superá-lo, a criação e o uso de métodos e tecnologias de ensino inovadoras e ativas são uma necessidade no contexto educacional. Busca-se, com eles, uma pedagogia democrática e transformadora ${ }^{(16)}$.

Diferentes benefícios advindos do uso de tecnologias no ensino foram percebidos por professores e docentes no decurso do período letivo, assim como nos processos avaliativos. Muitos deles já foram relatados pela literatura, como a potencialização do desenvolvimento de competências para atuação na enfermagem, evolução das habilidades manuais, autoconfiança nas atividades, comportamento ético, habilidade com instrumentos, conhecimento sobre o ambiente de trabalho, apreensão de novos saberes, melhor articulação teórico-prática e aumento da sensação de segurança ${ }^{(17-18)}$.

No campo da saúde, como aponta este relato, a conjugação de diferentes tecnologias mobiliza diferentes competências atitudinais, cognitivas, técnicas e éticas, tão necessárias aos enfermeiros, de modo que atuem com base na integralidade e 
humanização do cuidado ${ }^{(19)}$, isto é, possibilitem a produção de um trabalho vivo em ato, como ensina Merhy ${ }^{(6)}$. Pode-se, com base nessas tecnologias, superar também o modelo de atenção à saúde centrado meramente nos equipamentos e protocolos.

Espera-se que as aptidões e os saberes construídos durante a disciplina de semiologia e semiotécnica repercutam nos períodos posteriores do curso de graduação cujos temas, mais complexos, demandam maior capacidade intelectual para a interpretação dos dados objetivos e subjetivos, sem a qual o PE não se concretiza. De igual maneira, almeja-se que os conhecimentos produzidos coletivamente, de forma relacional entre docentes, monitores e alunos, sejam potencializadores de práticas efetivas de cuidado.

Como limitação do estudo, pontua-se que não foram empregados métodos de avaliação antes e depois da experiência relatada que permitissem a aferição do impacto do uso dessas tecnologias no ensino. Contudo, confia-se que este relato poderá servir de apoio metodológico para outras disciplinas, em distintos locais de ensino, assim como poderá subsidiar novos estudos que investiguem os efeitos dessa práxis pedagógica por meio de instrumentos metodológicos.

\section{Conclusão}

Este relato de experiência permite afirmar que o ensino de semiologia e semiotécnica em enfermagem pode fazer uso de diferentes tecnologias mediadoras do aprendizado, para favorecer o desenvolvimento das habilidades necessárias a uma atuação profissional centrada no usuário, na perspectiva de um processo de trabalho que se constrói com e para o usuário, sem perder de vista as sistemáticas que orientam o processo de trabalho do enfermeiro.

A disciplina objeto deste relato foi desenvolvida considerando que diferentes ambientes e pessoas exigem distintas abordagens que se adéquem à necessidade de aprendizagem de cada estudante. Também se adotou como pressuposto que o trabalho em enfermagem é sempre relacional, mesmo quando da execução de um ato técnico, o que requer dos enfermeiros competências interpessoais que, articuladas a outras competências, catalisarão a produção de novos modos de cuidar.

Depreende-se que o uso de diferentes tecnologias - leves, leve-duras e duras - no ensino de semiologia e semiotécnica de enfermagem favorece o desenvolvimento de habilidades e competências relacionais, manuais, éticas e humanísticas e fomenta a autonomia discente e o pensamento crítico-reflexivo necessários à articulação entre teoria e prática, à tomada de decisão clínica e aos novos modos de produzir cuidado em enfermagem.

\section{Colaborações:}

1 - concepção, projeto, análise e interpretação dos dados: Evandro Bernardino Mendes de Melo, Filipe Martinuzo Filetti, Lorena Barros Furieri e Mirian Fioresi;

2 - redação do artigo e revisão crítica relevante do conteúdo intelectual: Evandro Bernardino Mendes de Melo, Marcio Coutinho Farias Leite, Elizabete Regina Araújo de Oliveira, Lorena Barros Furieri e Mirian Fioresi;

3 - aprovação final da versão a ser publicada: Evandro Bernardino Mendes de Melo e Mirian Fioresi.

\section{Referências}

1. Kermansaravi F, Navidian A, Yaghoubinia F. Nursing students' views of nursing education quality: a qualitative study. Glob J Health Sci. 2015;7(2):351-9. DOI: 10.5539/ gjhs.v7n2p351

2. Melo GSM, Tibúrcio MP, Freitas CCS, Vasconcelos QLDAQ, Costa IKF, Torres GV. Semiotics and semiology of Nursing: evaluation of undergraduate students' knowledge on procedures. Rev bras enferm. 2017;70(2):249-56. DOI: 10.1590/0034-7167-2016-0417

3. Santos N, Veiga P, Andrade R. Importância da anamnese e do exame físico para o cuidado do enfermeiro. Rev bras enferm. 2011;64(2):355-8. DOI: $10.1590 /$ S0034-71672011000200021 
4. Santiago LC. O ensino da semiologia na graduação em enfermagem Rev Min Enferm. [Internet]. 2012 [cited 2019 Sep 12];16(4):485-6. Disponível em: http://www.reme.org.br/artigo/detalhes/551

5. Fernandes NC, Cunha RR, Brandão AF, Cunha LL, Barbosa PD, Silva CO, et al. Academic mentoring and care for a person with a stoma: experience report. Rev Min Enferm. 2015;19(2):238-41. DOI: $10.5935 / 1415-2762.20150038$

6. Merhy EE. Saúde: a cartografia do trabalho vivo. 2a ed. São Paulo: Hucitec; 2005.

7. NANDAInternacional. Diagnósticosdeenfermagem da NANDA-I: definições e classificação 2018-2020. 11a ed. Porto Alegre: Artmed; 2018.

8. Dochterman MCJ, Bulechek GM. Classificação das intervenções de enfermagem (NIC). 4 a ed. Porto Alegre: Artmed; 2008.

9. Horta WA. Processo de enfermagem. São Paulo: Pedagógica e Universitária; 1979.

10. Macedo ILJ, Zamarioli CM, Carvalho EC. Critical incidents involving Semiology during practical activities of nursing undergraduates. Rev bras enferm. 2018;71(Suppl 4):1572-9. DOI: 10.1590/0034-7167-2017-0364

11. Mattos MP, Gomes DR, Aleluia IRS, Sousa MLT. Promoção a saúde de estudantes universitários: contribuições para um espaço de integração e acolhimento. Saúde Redes. 2018;4(4):159-73. DOI: https://doi.org/10.18310/ 2446-48132018v4n4.2214g332

12. Andrade R, Ruoff AB, Piccoli T, Schmitt MD, Ferreira A, Xavier ACA. Case Study as a nursing research method: a integrative review. Texto Contexto Enferm. 2017;26(4):e5360016. DOI: 10.1590/0104-07072017005360016

13. Cogo ALP, Pai DD, Aliti GB, Hoefel HK, Azzolin KO, Busin L, et al. Case studies and role play: learning strategies in nursing.
Rev bras enferm. 2016;69(6):1231-5. DOI: 10.1590/0034-7167-2016-0277

14. Belayrla CJ, Gislaine FR, Cristiane CRS, Virginia COG, Gilberto TRS. Simulação em manequins como estratégia de ensino-aprendizagem para avaliação de ferida: relato de experiência. ESTIMA. 2017;15(4):245-9. DOI: 10.5327/Z1806-3144201700040009

15. Yamane MT, Machado VK, Osternack KT, Mello RG. Simulação realística como ferramenta de ensino na saúde: uma revisão integrativa. Espaç Saúde. 2019;20(1):87-107. DOI: 10.22421/ 15177130-2019v20n1p87

16. Simon E, Jezine E, Vasconcelos EM, Ribeiro KSQS. Metodologias ativas de ensino-aprendizagem e educação popular: encontros e desencontros no contexto da formação dos profissionais de saúde. Interface. 2014;18(Suppl 2):1355-64. DOI: $10.1590 / 1807-57622013.0477$

17. Vargas I, González X, Navarrete T. Metodología activa en el Estudio de Caso para desarrollo del pensamiento crítico y sentido ético. Enferm Univ. 2018;15(3):244-54. DOI: 10.22201/eneo.23958421e. 2018.3.65988

18. Dalcól C, Garanhani ML, Fonseca LF, Carvalho BG. Communication skills and teachinglearning strategies: perception of nursing students. Cogitare Enferm. 2018;23(3):e53743. DOI: 10.5380/ ce.v23i3.53743

19. Marinho PML, Campos MPA, Rodrigues EOL, Gis CFL, Barreto IDC. Construction and validation of a tool to Assess the Use of Light Technologies at Intensive Care Units. Rev Latino-Am Enfermagem. 2016;24:e2816. DOI: 10.1590/1518-8345.1002.2816

Recebido: 14 de outubro de 2019

Aprovado: 12 de novembro de 2019

Publicado: 11 de março de 2020

A Revista Baiana de Enfermagem utiliza a Licença Creative Commons - Atribuição-NãoComercial 4.0 Internacional. https://creativecommons.org/licenses/by-nc/4.0/

Este artigo é de acesso aberto distribuído sob os termos da Licença Creative Commons (CC BY-NC). Esta licença permite que outros remixem, adaptem e criem a partir do seu trabalho para fins não comerciais. Embora os novos trabalhos tenham de lhe atribuir o devido crédito e não possam ser usados para fins comerciais, os usuários não têm de licenciar esses trabalhos derivados sob os mesmos termos. 